\title{
A nonparametric CUSUM control chart based on the Mann-Whitney statistic
}

\author{
Dabuxilatu Wang* Qiang Xiong \\ Department of Statistics, School of Economics \\ and Statistics, Guangzhou University, No. 230 WaiHuan XiLu , \\ Higher Education Mega Center, Guangzhou, 510006, P.R.China
}

\begin{abstract}
This article aims to consider a new univariate nonparametric cumulative sum (CUSUM) control chart for small shift of location based on both change-point model and Mann-Whitney statistic. Some comparisons on the performances of the proposed chart with other charts as well as the properties of the test statistic are presented. Simulations indicate that the proposed chart is sensitive in detection of the small mean shifts of the process by a high intensive accumulation of sample information when the underlying variable is completely distribution-free.
\end{abstract}

keywords: Change-point, Mann-Whitney statistic, cumulative sum chart Mathematics Subject Classification (2000) 62p30

\section{Introduction}

Statistical process control (SPC) has been applied widely for monitoring various industrial manufacturing processes, service processes and some special behavior processes (see. Wetherill B. et al. [1], Montgomery [10]), in which control charts

\footnotetext{
${ }^{*}$ Corresponding author. E-mail:wangdabu@gzhu.edu.cn
} 
are the most widely used to detect changes in production process. In conventional $\mathrm{SPC}$, the monitored process variable is assumed to be modelled by the normal distribution, and based on such underlying distribution assumption, Shewhart chart, EWMA (exponential weighted moving average) chart and CUSUM (cumulative sum ) chart for variables data, p-chart or c-chart for attributes data, had been proposed (see. Wetherill B. et al. [1]). It is well recognized however that in many applications the underlying process distribution is not known (e.g. in phase I of SPC) sufficiently to assume normality (or any other parametric distribution), so that statistical properties of commonly used charts, designed to perform best under the assumed distribution, could be highly affected. In situation like this, development and application of control charts that do not depend on any specific parametric distributional assumptions, seem highly desired. There are literatures considering distribution-free charts or nonparametric charts for this purpose, an extensive overview on univariate nonparametric control charts was presented by Chakraborti [3]. While the average charts are probably the most widely used in detection of large mean shift because of their simplicity, CUSUM procedures are quite appropriate in view of the sequential nature of the process problem and more sensitive to small shift in process mean. Based on the within group Wilcoxon signed-rank statistic, Bakir and Reynold [2] proposed a nonparametric CUSUM chart to track the shift of a location parameter $\mu$ from an in-control known value $\mu_{0}$. McDonald [9] established some nonparametric CUSUM chart for detecting the process mean shifts by using sequential rank test. Using cross-sectional antiranks of the measurement as well as the order information of the sample, Qiu [12] [13] designed some nonparametric multivariate CUSUM control charts for detecting process variability and process mean shifts, respectively. Recently, in the case of having historical in-control data, Zhou et al. [16] established a nonparametric EWMA control chart by using the Mann-Whitney statistic, which performed a lower sensitivity than that of change point charts (see. Hawkins et al.[6]) having known normal distribution, and a higher sensitivity and robustness than that of change point charts (see. Hawkins et al.[6]) under non-normal distribution or 
distribution-free conditions. Chakraborti [4] also proposed a nonparametric average control chart-MW chart based on the Mann-Whitney statistic under condition of available reference sample from the in control process by a phase I analysis, which has a high sensitivity in detection of the large process mean shifts in a completely distribution-free case. Das [5] presented a note on efficiency of nonparametric control chart for monitoring process variability by using the rank-sum statistic of Ansari and Bradley. Yang and Cheng [15] proposed a nonparametric CUSUM mean chart based on the total number of univariate data exceeding the in-control mean which is already known or estimated by the available in control reference sample.

It is well known that the Mann-Whitney statistic is equivalent to the Wilcoxon rank-sum statistic. Though the rank based CUSUM charts have been proposed, there is no report on a nonparametric CUSUM chart based on the Mann-Whitney statistic. In this research, we design such CUSUM chart under the condition of having available in control reference sample like the cases of Zhou et al.[16], Chakraborti [4] and Yang and Cheng [15], and a finite sequence of future observations with considering the concept of change-point for detection of small shifts of the process mean (the location parameter). Some performance comparisons with other change point chart and nonparametric charts are considered. The rest of the paper is organized as follow. In Section 2, the concept of change-point and the Mann-Whitney statistic have been recalled. In Section 3, the test statistic of a nonparametric CUSUM chart based on the Mann-Whitney statistic is proposed, and the relevant control limits and control rule are designed. In Section 4, some performance comparisons of the designed chart with other charts are given. 


\section{Change-point and the Mann-Whitney statistic}

The traditional change-point model can be illustrated as follows. Let $\left\{X_{i}, i=\right.$ $1,2, \cdots\}$ be a sequence of independent random variables, and

$$
X_{i} \sim \begin{cases}F\left(x ; \mu_{0}, \sigma_{0}^{2}\right), & i=1,2, \cdots, \tau \\ F\left(x ; \mu_{1}, \sigma_{1}^{2}\right), & i=\tau+1, \tau+2, \cdots\end{cases}
$$

where $F$ stands for a continuous distribution function with unknown types, $\mu_{0}, \mu_{1}$ stand for the process mean and $\sigma_{0}^{2}, \sigma_{1}^{2}$ the process variance. If it holds $\mu_{0} \neq \mu_{1}$ or $\sigma_{0}^{2} \neq \sigma_{1}^{2}$, then $\tau$ is said to be a change-point. Detecting and finding out a change-point for random process is an important issue since with which we can predict properly the potential change in the observed process. The detection of the mean shifts or variance change in process control is similar to the detection of change-point in a process. Like Zhou et al. [16] we also consider a changepoint model based on sequence of finite independent random variables $\left\{X_{i}\right\}$, where $i=1,2, \cdots, l$.

In the nonparametric data analysis, a sort of change-point detection method is the well-known Mann-Whitney two-sample test (see. Mann and Whitney [8]), which is applied for inferring whether differences exist between the distributions of two populations through two independent group samples. For any $1 \leqslant t<l$, the Mann-Whitney statistic is defined as

$$
M W_{t, l}=\sum_{i=1}^{t} \sum_{j=t+1}^{l} I\left(x_{j}<x_{i}\right)=\sum_{j=t+1}^{l} I\left(x_{j}<x_{1}\right)+I\left(x_{j}<x_{2}\right)+\cdots+I\left(x_{j}<x_{t}\right),
$$

where

$$
I\left(x_{j}<x_{i}\right)= \begin{cases}1, & x_{j}<x_{i} \\ 0, & x_{j} \geqslant x_{i}\end{cases}
$$

If $\mu_{0}=\mu_{1}, \sigma_{0}^{2}=\sigma_{1}^{2}$, then it is said that the process is in-control state. In this paper, we only consider the variation on the location parameter and let $\sigma_{0}^{2}=\sigma_{1}^{2}$. It is verified that (see. Mann and Whitney [8], Zhou et al.[16]), under in-control state, the expectation and variance of $M W_{t, l}$ can be obtained as

$$
E_{0}\left(M W_{t, l}\right)=\frac{t(l-t)}{2}, \operatorname{Var}_{0}\left(M W_{t, l}\right)=\frac{t(l-t)(l+1)}{12}
$$


The standardized Mann-Whitney statistic $S M W_{t, l}$ is defined by

$$
S M W_{t, l}=\frac{M W_{t, l}-E_{0}\left(M W_{t, l}\right)}{\sqrt{\operatorname{Var}_{0}\left(M W_{t, l}\right)}} .
$$

Based on the proof of Mann-Whitney (see. Mann and Whitney [8]), when the process is in-control state, the distribution of $S M W_{t, l}$ is symmetric about zero for each $t$, and large values of $S M W_{t, l}$ indicate a negative mean shift, whereas small values indicate a positive shift [16]. As explained in Zhou et al. [16], a test statistic for detection of change-point about the mean (i.e. the hypotheses $H_{0}: \mu_{0}=\mu_{1}$ ) is proposed by Pettitt [11] as

$$
T_{l}=\max _{1 \leqslant t \leqslant l-1}\left|S M W_{t, l}\right|
$$

If $T_{l}$ exceeds some critical value $h_{l}$, then we conclude that there is a shift in the mean. Otherwise, we conclude that there is no sufficient evidence of a shift. To find a suitable critical values $h_{l}$, we can use the limiting distribution of $T_{l}$ given by Pettitt [1] to make an approximation.

Note that for each $t, S M W_{t, l}$ can be modeled approximately by standard normal distribution $N(0,1)$ when $l$ is large.

\section{A nonparametric CUSUM chart}

For detecting a small mean shift occurred at the change-point of the process as soon as possible, CUSUM control chart is usually recommended. Assuming that the occurred change-point indicates an upward mean shift, i.e., $\mu_{1}>\mu_{0}$, then at the change-point, the expectation of the Mann-Whitney statistic becomes larger and the sample mean around the change-point might occurs a small upward shift. In such case, it is hard to find shift quickly by using the statistic $T_{l}$, because it only depends on the finite individual observations without considering all historical sample information. Therefore, we desire to construct an upper side nonparametric CUSUM control chart based on the standardized Mann-Whitney statistic $S M W_{t, l}$.

Zhou et al. [16] have done an improvement on statistic $T_{l}$ and proposed the $S M W$ chart, which has a test statistic $T_{m, n}=\max _{m \leqslant t<m+n}\left|S M W_{t,(m+n)}\right|$. Fur- 
thermore, an EWMA chart has been given based on $S M W_{t,(m+n)}$, where $m$ is the number of in-control historical individual observations, $n$ is the number of the future observations, and $m+n$ is the number of the total observations. Noting that here the CUSUM chart has not been considered. Though both CUSUM chart and EWMA chart performed quite well in the detection of the small shifts in mean (see. Lucas and Saccucci [7]), CUSUM chart is usually slightly more sensitive than EWMA chart when the average in control run length become large (see. Srivastava and $\mathrm{Wu}$ [14]). Under the same condition of the $S M W$ chart, we propose the test statistic for our upper side CUSUM chart as

$S_{j}(m, n)=\max \left\{0, S_{j-1}(m, n)+S M W_{j,(m+n)}-k\right\}, j=m-m_{0}, m-m_{0}+1, \cdots, m-m_{0}+n-1$,

where $0 \leqslant m_{0} \leqslant m, S_{m-m_{0}-1}(m, n)=0, k=\frac{\Delta}{2}$ is the reference value, $\Delta$ is the shift size to be detected. Here we assume $k=\frac{1}{2}$. Set

$$
S_{\text {max }}(m, n)=\max _{m-m_{0} \leqslant j \leqslant m+n-1}\left|S_{j}(m, n)\right|=\max _{m-m 0 \leqslant j \leqslant m+n-1} S_{j}(m, n) .
$$

Our control rule is as follow,

(1)After the $n$th future sample is monitored, compute $S_{\max }(m, n)$.

(2)Let $h_{m, n}$ be the decision value, which is chosen to obtain the given in-control average run length. If $S_{\max }(m, n) \leqslant h_{m, n}$, we conclude that there is no evidence of a shift and continue to monitor the $(n+1)$ st future sample. If $S_{\max }(m, n) \geqslant h_{m, n}$, then an out-of-control signal is triggered.

Noting that, in the case of SMW chart (see. Zhou et al. [16]), we need to calculate the maximum values of $S M W_{t,(m+n)}$ for each $t$, whereas for our CUSUM chart, we calculate the maximum values of the cumulative sum $S_{j}(m, n)$.

For the given type one error $\alpha$, the decision value $h_{m, n}$ can be obtained by solving following equations

$$
\begin{gathered}
\operatorname{Pr}\left(S_{\max }(m, n)>h_{m, n}(\alpha) \mid S_{\max }(m, i) \leqslant h_{m, i}(\alpha), 1 \leqslant i<n\right)=\alpha, n>1 \\
\operatorname{Pr}\left(S_{\max }(m, 1)>h_{m, 1}(\alpha)\right)=\alpha .
\end{gathered}
$$

Due to the intricacy of this conditional probability, it seems to be impossible to solve it analytically. Therefore, similar to [16], we use one million sequences of 
length 500 which come from the standard normal distribution to estimate them. The historical sample size is assumed to be larger than 10. Table 1 shows the control limit of CUSUM chart for $\alpha$ values of 0.01, 0.005, 0.0027, and 0.002, corresponding to in control ARLs of $100,200,370,500$, for $m=10$ and 50, $m_{0}=4$, and $n$ values in the range $1-490$. As shown in Table $1, h_{m, n}(\alpha)$ increase initially, but then stabilizes. We can obtain the optimal decision value using such approach of estimation. Compare with the decision values $h_{m, n}(\alpha)$ shown in Table 1 of Zhou et al. 16] for their EWMA chart, the decision values of CUSUM chart seem slightly smaller. Similar to Zhou et al.[16], it is not difficult to present an illustrative example to introduce the implementation of our proposed CUSUM chart, we omit it here.

The equivalence between the Wilcoxon rank-sum statistic $W_{t, l}$ and the MannWhitney statistic $M W_{t, l}$ is shown with the equality $M W_{t, l}=W_{t, l}-\frac{t(t+1)}{2}$, where $W_{t, l}=\sum_{i=1}^{t} R_{i}$, and $R_{i}$ denotes the rank of the $i$ th observation $x_{i}$ in the total $l$ observations. We may use this equivalence to reduce the computational complexity of the Mann-Whitney statistic.

\section{A comparison between control charts}

In this section, we present a simple performance comparison shown in Table 2 between our CUSUM chart and the change-point chart proposed by Hawkins et al. [6] based on the available data from Zhou et al. [16]. Also we consider some comparisons of the characteristics of our test statistic to other nonparametric CUSUM statistic.

\subsection{A comparison with change-point chart}

In Table $2, \delta$ denotes the coefficient of standard deviation for measuring the shift size, $\tau$ denotes the change-point. We can found that

(1) As the increasing of the future observed in-control data, both charts become more sensitive to the shift as the new observation updates the information already 
Table 1

The decision values $\left.h_{(} m, n\right)(\alpha)$ of CUSUM control chart, $k=\frac{1}{2}$

\begin{tabular}{|c|c|c|c|c|c|c|c|c|}
\hline & $\mathrm{m}=10$ & & & & $\mathrm{~m}=50$ & & & \\
\hline & $\operatorname{ARL}(0)$ & & & & & & & \\
\hline $\mathrm{n}$ & 100 & 200 & 370 & 500 & 100 & 200 & 370 & 500 \\
\hline 1 & 1.120 & 1.185 & 1.184 & 1.114 & 1.182 & 1.192 & 1.235 & 1.254 \\
\hline 3 & 1.263 & 1.329 & 1.216 & 1.412 & 1.346 & 1.395 & 1.459 & 1.502 \\
\hline 5 & 1.462 & 1.589 & 1.668 & 1.693 & 1.554 & 1.660 & 1.748 & 1.827 \\
\hline 7 & 1.611 & 1.752 & 1.865 & 1.914 & 1.696 & 1.847 & 1.974 & 2.038 \\
\hline 9 & 1.689 & 1.845 & 1.972 & 2.021 & 1.774 & 1.945 & 2.082 & 2.150 \\
\hline 11 & 1.744 & 1.916 & 2.050 & 2.119 & 1.833 & 2.023 & 2.160 & 2.238 \\
\hline 13 & 1.798 & 1.988 & 2.129 & 2.167 & 1.887 & 2.082 & 2.238 & 2.316 \\
\hline 15 & 1.836 & 2.027 & 2.178 & 2.246 & 1.996 & 2.121 & 2.297 & 2.375 \\
\hline 17 & 1.873 & 2.076 & 2.227 & 2.295 & 1.950 & 2.160 & 2.336 & 2.484 \\
\hline 19 & 1.897 & 2.090 & 2.261 & 2.334 & 1.970 & 2.180 & 2.360 & 2.453 \\
\hline 22 & 1.924 & 2.129 & 2.295 & 2.393 & 1.989 & 2.198 & 2.394 & 2.472 \\
\hline 26 & 1.951 & 2.170 & 2.349 & 2.432 & 1.999 & 2.238 & 2.434 & 2.522 \\
\hline 30 & 1.985 & 2.191 & 2.383 & 2.481 & 2.038 & 2.192 & 2.453 & 2.551 \\
\hline 35 & 2,065 & 2.237 & 2.422 & 2.510 & 2.043 & 2.297 & 2.483 & 2.581 \\
\hline 40 & 2.026 & 2.268 & 2.452 & 2.549 & 2.063 & 2.367 & 2.502 & 2.60 \\
\hline 50 & 2.049 & 2.292 & 2.496 & 2.588 & 2.078 & 2.327 & 2.522 & 2.620 \\
\hline 60 & 2.071 & 2.317 & 2.530 & 2.628 & 2.097 & 2.346 & 2.552 & 2.649 \\
\hline 70 & 2.083 & 2.333 & 2.546 & 2.638 & 2.107 & 2.356 & 2.571 & 2.669 \\
\hline 80 & 2.091 & 1.341 & 2.559 & 2.657 & 2.112 & 2.366 & 2.591 & 2.688 \\
\hline 90 & 2.095 & 2.356 & 2.569 & 2.667 & 2.117 & 2.386 & 2.60 & 2.698 \\
\hline 115 & 2.106 & 2.372 & 2.589 & 2.686 & 2.122 & 2.386 & 2.605 & 2.708 \\
\hline 140 & 2.118 & 2.380 & 2.60 & 2.6906 & 2.127 & 2.390 & 2.610 & 2.718 \\
\hline 165 & 2.152 & 2.388 & 2.608 & 2.706 & 2.129 & 2.395 & 2.655 & 2.727 \\
\hline 190 & 2.136 & 2.405 & 2.628 & 2.726 & 2.142 & 2.415 & $2.63 \mathrm{o}$ & 2.742 \\
\hline 240 & 2.134 & 2.413 & 2.633 & 2.735 & 2.147 & 2.420 & 2.635 & 2.747 \\
\hline 290 & & 2.421 & 2.643 & 2.745 & & 2.425 & 2.620 & 2.752 \\
\hline 390 & & & 2.658 & 2.760 & & & 2.659 & 2.767 \\
\hline 490 & & & & 2.795 & & & & 2.800 \\
\hline
\end{tabular}


Table 2

The ARL comparisons between CUSUM chart and C-PC (Change-Point Chart)

for $\mathrm{N}(0,1)$ data and $\mathrm{m}=10, \alpha=0.005$

\begin{tabular}{|l|l|l|l|l|l|l|l|l|}
\hline & $\tau=10$ & & $\tau=50$ & & $\tau=100$ & & $\tau=250$ & \\
\hline$\delta$ & CUSUM & C-PC & CUSUM & C-PC & CUSUM & C-PC & CUSUM & C-PC \\
\hline 0.00 & 200.0 & 200.0 & 200.0 & 200.0 & 200.0 & 200.0 & 200.0 & 200.0 \\
0.025 & 173.1 & 187.5 & 123.4 & 155.5 & 96.4 & 130.7 & 70.8 & 100.3 \\
0.050 & 134.8 & 159.8 & 39.9 & 66.4 & 26.8 & 41.1 & 21.9 & 31.1 \\
0.75 & 92.1 & 113.5 & 13.9 & 22.8 & 11.9 & 17.0 & 10.9 & 15.1 \\
1.00 & 52.80 & 65.5 & 8.7 & 11.6 & 8.2 & 9.9 & 8.2 & 9.2 \\
1.25 & 26.6 & 30.6 & 6.9 & 7.5 & 6.7 & 6.7 & 6.9 & 6.4 \\
1.50 & 14.2 & 15.0 & 6.3 & 5.4 & 5.9 & 5.0 & 5.8 & 4.8 \\
1.75 & 8.2 & 8.8 & 5.2 & 4.2 & 5.3 & 3.9 & 5.1 & 3.7 \\
2.00 & 5.8 & 6.3 & 4.3 & 3.4 & 4.3 & 3.2 & 4.2 & 3.0 \\
2.25 & 5.4 & 5.0 & 3.9 & 2.8 & 3.9 & 2.6 & 3.9 & 2.6 \\
2.50 & 4.2 & 4.2 & 3.4 & 2.4 & 3.5 & 2.3 & 3.5 & 2.2 \\
2.75 & 3.8 & 3.6 & 3.1 & 2.1 & 3.1 & 2.0 & 2.9 & 1.9 \\
3.00 & 3.5 & 3.1 & 2.8 & 1.8 & 2.9 & 1.8 & 2.6 & 1.7 \\
\hline
\end{tabular}

known.

(2) For detecting a relatively large shift in mean, the change-point chart proposed by Hawkins et al. [6] is more sensitive than our CUSUM chart.

(3) Our CUSUM chart is faster than the change-point chart proposed by Hawkins et al. [6] for detecting a small mean shift.

Similar to Zhou et al. [16], we may make other performance comparisons of our CUSUM chart to other control charts having known underlying distribution, we omit it here.

\subsection{A comparison with other nonparametric CUSUM charts}

In the following, we also mention a rough comparison between our CUSUM chart and other nonparametric CUSUM charts proposed by Bakir and Reynolds [2], McDonald 9] and Yang and Cheng [15] based on the characteristics of the test statistic. 
Bakir and Reynolds [2] consider a sequence of observations $\left\{x_{i j}, i=1,2, \cdots ; j=\right.$ $1, \cdots, g$, each of size $g=4$ or 5 , and define a within group Wilcoxon signed rank sum $S R_{i}=\sum_{j=1}^{g} \operatorname{sign}\left(x_{i j}\right) R_{i j}$ for each observation, where $R_{i j}$ denotes the rank of $\left|x_{i j}\right|$ in $\left\{\left|x_{i 1}\right|, \cdots,\left|x_{i g}\right|\right\}$, and based on which they propose their CUSUM statistic as

or

$$
\sum_{i=1}^{n}\left(S R_{i}-k\right)-\min _{0 \leqslant m \leqslant n} \sum_{i=1}^{m}\left(S R_{i}-k\right),
$$

$$
\max _{0 \leqslant m \leqslant n} \sum_{i=1}^{m}\left(S R_{i}+k\right)-\sum_{i=1}^{n}\left(S R_{i}+k\right) .
$$

From the structure of the statistic, we are not able to compare it straightforwardly with our CUSUM statistic because the rank in Mann-Whitney is based on the comparison of the total samples, whereas the rank in former statistic is only based on comparison within group sample with its absolute values. If we restrict a sequence of group sample of size $g$ to finite $l$ times observations, then we may view them as a finite sequence of $l \times g$ independent random variables, and we may assume the former $m$ variables of the sequence are in-control state, so that we can obtain our standardized Mann-Whitney statistic with a reference in-control data. Noting that the rank involved in our CUSUM statistic is for total samples and the rank in Bakir and Reynolds 's CUSUM statistic [2] is only the within group signed rank, the sum of the former ranks is obviously larger than the sum of the later ranks. Therefore, for a fixed reference value $k$ and a decision value $h$, our CUSUM chart is clearly more sensitive than Bakir and Reynolds's chart for small mean shift.

For comparing our CUSUM chart with McDonald's CUSUM chart [9], we note that their sequential rank $R_{i}$ is defined as $R_{i}=1+\sum_{j=1}^{i-1} I\left(x_{j}<x_{i}\right)$ for the observation $\left\{x_{i}, i=2, \cdots\right\}$, and the CUSUM statistic $T_{j}=\max \left\{0, T_{j-1}+U_{j}-k\right\}$, where $U_{i}=\frac{R_{i}}{i+1}$. The Mann-Whitney statistic $M W_{i, n}$ can be written as

$$
M W_{i, n}=\left(R_{i+1}-1\right)+\sum_{s=i+2}^{n}\left(I\left\{x_{1}<x_{s}\right\}+I\left\{x_{2}<x_{s}\right\}+\cdots+I\left\{x_{i}<x_{s}\right\}\right),
$$

$i=2, \cdots, n-1$. Therefore, $M W_{i, n}$ is obviously larger than $R_{i}$, which leads to that the cumulative sample information of our CUSUM statistic is more rich than the 
cumulative sample information of $T_{i}$. So our CUSUM chart based on $M W_{i, n}$ is more sensitive than the CUSUM chart based on $R_{i}$ proposed by McDonald [9].

We now consider roughly to compare our CUSUM chart with Yang and Cheng's CUSUM chart [15]. Noting that their chart is based on statistic $M_{t}=\sum_{j=1}^{t} I\left\{x_{j}>\right.$ $\mu\}$ for the observations $\left\{x_{i}, i=1,2, \cdots\right\}$, where $\mu$ is the in control mean of the process. Obviously, the Mann-Whitney statistic $M W_{i, n}$ implies more information than $M_{t}$, especially in the case where the unknown in control mean need to be estimated by the available reference in-control samples. They only utilize the average information like $\overline{\bar{x}}$ of the available reference in-control samples, whereas in our CUSUM case, the $m$ available reference in control samples are fully utilized with an individually comparison. So our CUSUM chart is also relatively more sensitive than Yang and Cheng's CUSUM chart.

Conclusion remark The rank based statistical method is an important wellknown nonparametric approach for the case where the distribution of the underlying variable is completely unknown, in which the Mann-Whitney statistic is the popular and powerful one. We establish a sort of nonparametric CUSUM chart based on the standardized Mann-Whitney statistic for the detection of the small location shifts quickly. Comparisons indicate that the proposed CUSUM chart is slightly quicker than the nonparametric charts proposed by Zhou et al. [16], Bakir and Reynolds [2], McDonals [9] and Yang and Cheng [15] in the detection of the small mean shifts. However, the computation of our CUSUM statistic is somewhat difficult, we need to solve it with the computer programming. It is also to be pointed out that the nonparametric control charts is rest on the theoretical research only, of which the development of the practical applications is highly desired.

\section{References}

[1] Barrie Wetherill G. and Brown D. W., (1991), Statistical process control, Theory and practice, Chapman and Hall, London, New York, Toyko, Melbourne, Madras, 
[2] Bakir, S.T. and Reynolds, M.R.JR , (1979), A nonparametric procedure for process control based on within group ranking, Technometrics, 21: 175-183.

[3] Chakraborti S., Van Der Laan P. and Bakir S.T., (2001), Nonparametric control charts: An Overview and Some Results, Journal of quality technology, 33 (3): 304-315.

[4] Chakraborti S. and Van De Wiel Mark A., (2008), A nonparametric control chart based on the Mann-Whiteney statistic, IMS collections, beyond parametrics in interdisciplinary research: festschrift in honor of profesor Pranab K. Sen, Vol 1: 156-172.

[5] Das, N., (2008), A note on the efficiency of nonparametric control chart for mornitoring process variability, Economic quality control, 23(1): 85-93.

[6] Hawkins, D.M., Qiu, P., Kang, C.W., (2003), A changepoint model for statistical process control. J Qual. Technol. 35: 355-366.

[7] Lucas,J.M., Saccucci, M.S., (1990), Exponentially weighted moving average control charts schemes: properties and enhancements, Technometrics, 32:1-30.

[8] Mann H.B., Whitney D.R., (1947), On the test whether one of two random variables is stochastically larger than other. Ann. Math. Stat. 18:50-60.

[9] McDonald, D., (1990), A CUSUM procedure based on sequential ranks, Naval research logistics, 37: 627-646.

[10] Montgomery, D.C., (2004), Introduction to statistical quality control, 5th edition. New York: Wiley.

[11] Pettitt A.N., (1979), A non-parametric approach to the change-point problem. Applied Statistics. 28: 126-135.

[12] Qiu, P., Hawkins, D.M., (2001), A rank based multivariate CUSUM procedure, Technometrics, 43: 120-132. 
[13] Qiu, P., Hawkins, D.M., (2003), A nonparametric multivariate CUSUM procedure for detecting shifts in all directions, Statistician, 52: 152-164.

[14] Srivastava, M.S., Wu, Y., (1993), Comparison of EWMA, CUSUM and Shiryayev-Roberts procedures for detecting a shift in the mean, The annals of statistics, 21(2): 645-670.

[15] Su-fen Yang and Smiley W. Cheng, (2010), A new nonparametric CUSUM mean chart, Quality and reliability engineering international, doi: 10.1002/qre.1171.

[16] Zhou Chunguang, Zou Changliang, Zhang Yujuan, Wang Zhaojun. (2009), Nonparametric control chart based on change-point model. Statistical Papers. 50:13-28. 